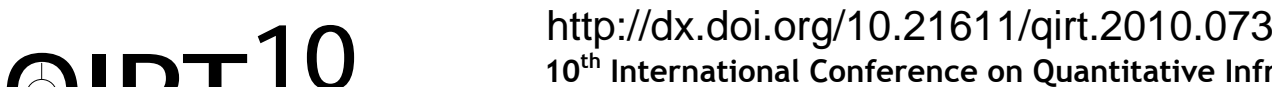 \\ $10^{\text {th }}$ International Conference on Quantitative InfraRed Thermography \\ July 27-30, 2010, Québec (Canada)
}

\section{Quantitative dual-spectrum infrared approach for breast cancer detection}

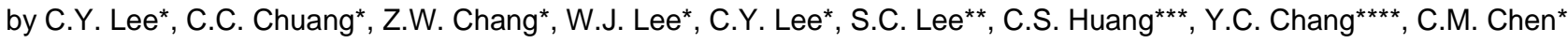 \\ *BME Inst., Coll. of Med. and Coll. of Eng., National Taiwan University, Taipei, Taiwan106, Olive.ntu@gmail.com \\ **EE Dept., National Taiwan University, Taipei, Taiwan10617, sclee@cc.ee.ntu.edu.tw \\ ***Surgery Dept., Coll. of Med., National Taiwan University, Taipei, Taiwan106, huangcs@ntu.edu.tw \\ ${ }^{\star * \star R}$ Radiology Dept., Coll. of Med., National Taiwan University, Taipei, Taiwan106, ycc5566@ntu.edu.tw
}

\begin{abstract}
Infra-red images for chemotherapy assessment of breast lesions remain controversial because of physiological and environmental factors. To minimize these effects, a quantitative dual-spectrum IR system is proposed. The idea is to use middle-wave and long-wave IR information to measure the heat pattern change on the breast skin. An algorithm is developed to separate the tissue areas of high and normal temperature. Two descriptors are computed to indicate the structural and functional change of the tissues. The preliminary results show both descriptors tend to decrease as the patients receive more chemotherapy, suggesting the proposed system has the potential for chemotherapy assessment.
\end{abstract}

\section{Introduction}

Serving as a medical imaging modality, the Infra-red (IR) image reveals the heat distribution on the surface of the human body. Cancerous tissues tend to have a higher temperature signature than their surrounding normal tissues, for this reason, IR image has long been studied in hope to serve as indicator for cancerous breast tissues. Nevertheless, the usefulness of IR images in detecting breast cancers remains controversial due to the physiological and environmental influence on the skin temperature distribution, and most importantly, there are no objective methods to quantitatively analyze lesion malignancy.

Various approaches have been proposed in detecting breast cancers or assess treatment results of breast cancer chemotherapy using the IR image. These methods can be classified into two general categories, namely cross-sectional and longitudinal approaches, primarily rooted in two important findings. One is the well-known phenomenon that tumor growth is usually accompanied by angiogenesis leading to local vascularization. The other is the temperature of the breast area with tumors is generally higher than that of the normal tissue area [1].

The cross-sectional approaches are mainly based on the comparison between the normal area and the abnormal area of the breast. Many studies have made use of the symmetrical property existed in the blood vessel or temperature patterns of both breasts to evaluate the abnormality of a breast $[2,3]$. However, Cunningham had advocated that relying on the concept of vascular symmetry to determine the abnormality of a breast is a fallacy because the distribution of blood vessel along both sides of breast is generally unsymmetrical[4]. Moreover, the anatomy of human breast is very complicated. Different energy may be radiated due to different metabolism. Although the temperature of the breast area with tumors is expected to be higher than that of the normal breast area $[5,6]$, it does not necessarily mean that the high temperature area in IR images corresponds to the tumor region [7]. Therefore, it is too arbitrary to use the heat pattern acquired at a single time point as the basis to determine if there is a tumor in a breast.

Alternatively, the longitudinal approaches attempt to determine the malignancy of breast tissues based on the variation of heat pattern over several time points. The general idea is the heat pattern of each person remains roughly unchanged under similar physiological and environmental conditions. It has been observed that the heat patch in the IR image may shrink or even disappear in what seems like a direct correlation to the tumor blood vessels breaking down and eventually disappear as treatment progress [8]. To quantitatively analyze this subjective visual judgment on the change of a breast tumor, Ng. et al. divide each breast into four parts and compare the temperature distribution of each pair of corresponding parts [9]. The potential deficiency of this approach lies in the fallacious implicit assumption that the two breasts under the normal condition have the same temperature distributions.

Another problem that a longitudinal approach may have is the noisy variations caused by the physiological and environmental factors at different time points. Although the influence of such environmental variables as temperature, humidity, and so on, may be minimized through deliberate control, these factors could still disturb the IR intensity fluctuation and affects the quantitative estimation of IR intensity change along the time.

To detect and quantify the breast cancer information collected under minimal physiological and environmental effects, one possible solution is to employ multiple-spectrum IR information at the same time in a longitudinal approach. By combining different energies received in the IR images of different spectrums, this approach offers an opportunity to further reduce the influence of the physiological and environmental factors. One excellent pioneer work of this kind is the blind source separation algorithm developed by Szu et al [10]. Using two satellite-grade infrared cameras, Szu et al. demonstrated that it 
is likely to detect breast cancer based on a pair of MIR and LIR breast images. Since this algorithm[10] mainly counts on the minute difference between the vectors, i.e., (MIR, LIR), of the cancerous and normal tissues, further investigation is required to attain a sufficient signal-to-noise ratio for this idea to be applied in a clinical setting.

Rather than directly estimating the likelihood that each pixel contains cancerous tissue as in [10], a new longitudinal approach is proposed in this paper. Our basic idea is to estimate the heat pattern change on the breast skin with IR photon information from a pair of MIR and LIR cameras. To quantify the change of the heat pattern, a dual-spectrum heat pattern separation (DS-HPS) algorithm is developed to separate the tissue area of high temperature from that of normal temperature in both of the MIR and LIR images. Two descriptors are computed to indicate the structural and functional change of the tissues due to chemotherapy. One is the area of the high temperature region on the breast surface and the other is the mean effective volume of high temperature tissues under the high temperature region. Increasing or decreasing of these two descriptors may indicate the existence of malignant breast lesions and effectiveness of chemotherapy, respectively.

\section{METHOD}

\subsection{Spatiotemporal registration algorithm}

Images generate by medical modalities such as X-ray mammograms, MR images and Ultrasound has the characteristic of showing distinct anatomic features such as nipples and boundaries. These features are easy to distinguish such that they are used as feature points for the transformed models [11]. In contrast, infrared images have less distinct boundaries, therefore it is challenging to detect feature points for image registration and making infrared images alignment a difficult hurdle to cross. A spatiotemporal registration algorithm to quantify longitudinal infrared images is established and shown in figure 1 .

The registration model includes three stages: begin by detecting feature points, follow with searching the corresponding feature pairs, and lastly dynamical correct the corresponding point locations. The heat patterns in the normal tissue show less variation than in the abnormal tissue for longitudinal infrared images. According to this characteristic, feature point detections in normal tissue can be performed automatically via Harris corner detector. However, determining the corresponding relationship between feature points must be established manually. The corresponding feature pairs are used as data sets for TPS transformed model to perform image registration. The mutual information is used to compare and measure quantitatively how closely target images and registered source images matched. Applying these mutual information values through Nelder-Mead simplex method, relationship functions are adjusted to improve accuracy until the mutual information value is optimized within iterations. In this way, a more robust registered source image could be obtained.

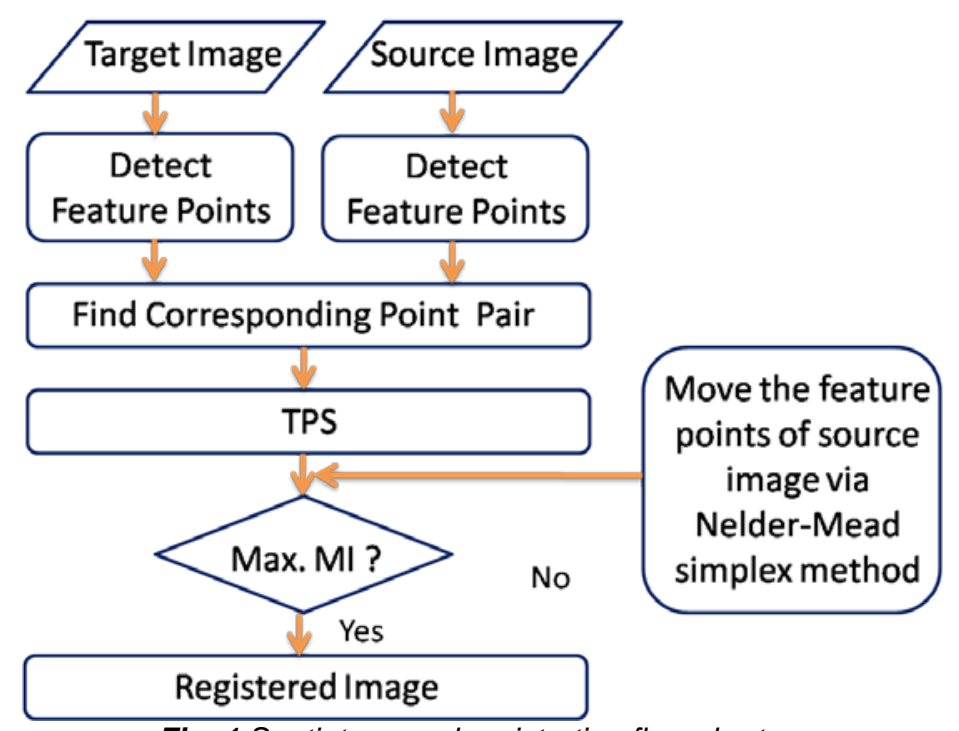

Fig. 1.Spatiotemporal registration flow chart

\subsection{Dual-Spectrum Heat Pattern Separation algorithm}

The DS-HPS algorithm aims to separate the high temperature region from the normal temperature region on the surface of both breasts of a subject. Since the cancerous tissues, including the malignant lesions and the vessels connected to the lesions, tend to have a higher temperature than the surrounding normal tissues, quantitative analysis of the change of the 
high temperature region may reveal the existence of malignant breast lesions and effectiveness of chemotherapy. The DSHPS algorithm is formulated as a blind source separation (BSS) algorithm. Let $\mathbf{x}=\left(x_{L}, x_{M}\right)$ be the energy reading vector of a corresponding point pair in the LIR and MIR images. Suppose $M_{N}$ and $M_{H}$ represents the energy detected by the MIR camera, emitted from each unit volume of the normal temperature tissue and the high temperature tissue, respectively. Similarly, suppose $L_{N}$ and $L_{H}$ represents the energy detected by the LIR camera, emitted from each unit volume of the normal temperature tissue and the high temperature tissue, respectively. Moreover, let $k_{N}$ and $k_{H}$ be the effective volume of the normal temperature tissue and the high temperature tissue, respectively. Note that the effective volume is not equivalent to the actual volume. The effective volume takes into account the energy attenuation during the propagation from the IR photon generation site to the breast surface.

The energies detected by the LIR and MIR cameras may be approximated as a linear composition of the energies of the attenuated LIR and MIR photons originated from the high temperature and normal temperature tissues as follows:

$$
\left[\begin{array}{c}
x_{L} \\
x_{M}
\end{array}\right]=\left[\begin{array}{cc}
L_{H} & L_{N} \\
M_{H} & M_{N}
\end{array}\right]\left[\begin{array}{c}
k_{H} \\
k_{N}
\end{array}\right]
$$

If we let $L_{H}=A_{H} \cos \theta, M_{H}=A_{H} \sin \theta, L_{N}=A_{N} \cos \phi$ and $M_{N}=A_{N} \sin \phi$, equation (1) can be represented as:

$$
\left[\begin{array}{l}
x_{L} \\
x_{M}
\end{array}\right]=\left[\begin{array}{ll}
\cos \theta & \cos \phi \\
\sin \theta & \sin \phi
\end{array}\right]\left[\begin{array}{l}
q_{H} \\
q_{N}
\end{array}\right]
$$

where $q_{H}=A_{H} k_{H}$ and $q_{N}=A_{N} k_{N} \cdot q_{H}$ and $q_{N}$ may be considered as the quantity approximately equivalent to the total energies of the attenuated LIR and MIR photons originated from the high temperature tissue and the normal temperature tissue, respectively.

There are 4 unknowns in equation (2) but with only 2 equations. To solve this dilemma, it is assumed that the parameter $\phi$ and $q_{N}$ change slowly in the normal temperature region. Based on these two assumptions, the cost function of this algorithm may be formulated as:

where

$$
f=A+B+C+D+\lambda\left[q_{N}-q_{N 0}\right]^{2}
$$

$$
\begin{gathered}
A=\left[X_{L}-\left(\cos \theta \cdot q_{H}+\cos \phi \cdot q_{N}\right)\right]^{2} \\
B=\left[X_{M}-\left(\sin \theta \cdot q_{H}+\sin \phi \cdot q_{N}\right)\right]^{2} \\
C=\alpha_{H}^{2}\left[\left(\frac{\partial q_{H}}{\partial x}\right)^{2}+\left(\frac{\partial q_{H}}{\partial y}\right)^{2}\right] \\
D=\alpha_{N}^{2}\left[\left(\frac{\partial q_{N}}{\partial x}\right)^{2}+\left(\frac{\partial q_{N}}{\partial y}\right)^{2}\right]
\end{gathered}
$$

$C$ and $D$ are the smoothness constraint. $q_{N_{0}}$ represents the initial $q_{N}$. $\lambda$ is the Lagrange multiplier.

By using the Newton-Raphson method, iterative equations for $q_{N}$ and $q_{H}$ may be derived as follows:

Where

$$
\begin{aligned}
& q_{N}^{n+1}=\frac{\{E \cdot \cos (\theta-\phi)\}-\left[F+\lambda q_{N_{0}}\right]\left(1+\alpha_{H}\right)}{G} \\
& q_{H}^{n+1}=\frac{\left.\left\{F+\lambda q_{N_{0}}\right] \cos (\theta-\phi)\right\}-E\left(1+\alpha_{N}+\lambda\right)}{G}
\end{aligned}
$$

$$
\begin{aligned}
& E=\left[\alpha_{H} \bar{q}_{H}+\left(X_{L} \cos \theta+X_{M} \sin \theta\right)\right] \\
& F=\alpha_{N} \bar{q}_{N}+\left(X_{L} \cos \phi+X_{M} \sin \phi\right) \\
& G=\left[\cos ^{2}(\theta-\phi)-\left(1+\alpha_{H}\right)\left(1+\alpha_{N}+\lambda\right)\right]
\end{aligned}
$$


To avoid being trapped in a local minimum, simulated annealing is used in collaboration with the Newton-Raphson method. Once the optimization process converges, $q_{H}$ map is thresholded in such a way that all values smaller than 0.001 are to 0 . The high temperature region is then defined by the remaining nonzero pixels in the $q_{H}$ map.

\subsection{Descriptors of High Temperature Region}

To quantitatively characterize the structural and functional change of the tissues caused by chemotherapy or tumor growth, two descriptors are computed from the high temperature region. The first one, denoted by HT-area, is the area of the high temperature region. The second one, denoted by HT-E, is the mean energy originated from the high temperature tissues and detected by the IR cameras. It is expected that both of HT-area and HT-E decrease as a patient receives more chemotherapy for an effective chemotherapeutic course.

\section{RESULT AND DISCUSSION}

Experimental results show that the proposed approach can effectively quantify the variation of the lesion size and its peripheral blood vessels in the course of chemotherapy. For example, figure 2 is a pair of original MIR and LIR images of a patient taken by the proposed quantitative dual-spectrum infrared (QDS-IR) system. The small red spots in these two images are markers for registration. On the right side of each IR image there is a color reference gauge for pseudo-coloring. Figures 3(a) and 3(b) give the MIR and LIR images taken at the same time, respectively, and figure 3(c) demonstrates the registered image via TPS method.

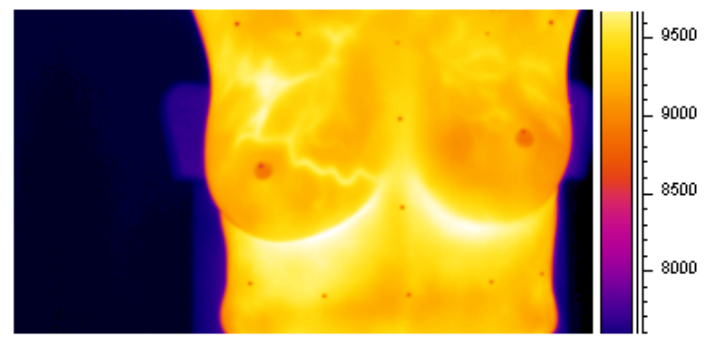

(a)

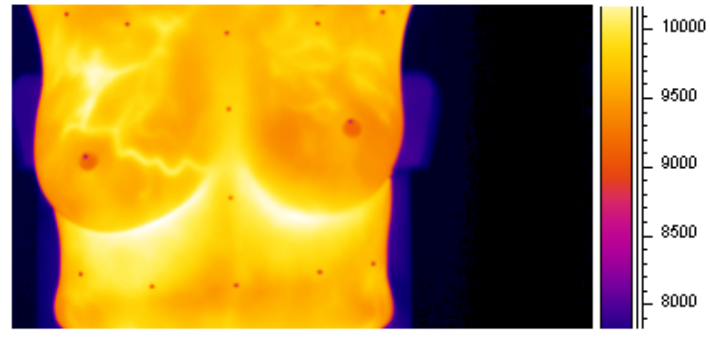

(b)

Fig. 2. (a) LIR image, (b) MIR image; taken by our quantitative dual-spectrum infrared system, in which the small red spots are markers for registration.

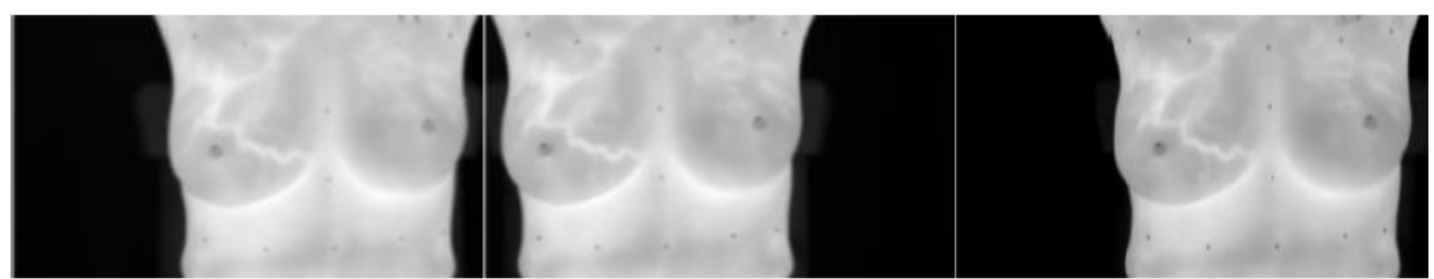

Fig. 3. (a) LIR image, (b) MIR image, (c) the TPS-transformed MIR image

It is possible to deduce the tumor location from the results. Figure 4(a) presents the results of the DS-HPS algorithm for 5 pairs of MIR and LIR images taken in a sequence of chemotherapy. Two dates are shown on top of each column. The date on the row "IR" is the date when IR images were taken, and "chemo" is the chemotherapy day. Columns from top to bottom represent ROIs and $q H$ maps respectively. If the effectiveness of the chemotherapy is assessed by observing only MIR or LIR images as shown in the ROI row of Figure 4(a), it is obviously difficult to determine the change of the lesion size and its peripheral vessels quantitatively. In addition, we cannot estimate the variation of energy emitted from the cancerous tissues. However, the $q H$ map in the last row of Figure 4(a) clearly shows the structural change of cancerous tissues as well as the variation of energy detected from cancerous tissues.

The two descriptors, i.e., HT-area and HT-E, are plotted in figure 4(b) and (c). HT-area shows a decreasing trend in the overall high temperature area, consistent with the reduced tumor size as confirmed by the MRI, Ultrasound and Mammogram. The tumor location is L11/4-12/3-1/4 (11:30 to 13:00 of the left breast, 3 to $4 \mathrm{~cm}$ from the nipple) and the tumor size is about $2 \mathrm{~cm} \times 4 \mathrm{~cm}$. After chemotherapy, the tumor size becomes $0.9 \mathrm{~cm} \times 3 \mathrm{~cm}$. Based on the HT-area result, five small ROIs are selected in each $q H$ map for further estimate the probability of tumor in these locations and assess the response to 
chemotherapy. Figure 4(c) shows the HT-E descriptor of five red areas. The HT-E curves of line 4 (purple) and line 5 (light blue) show an obvious decreasing trend, consistent with the reduced tumor size as confirmed by the MRI result. It could be inferred that there might be tumors in the 4th and 5th locations of the infrared image due to energy decrease after chemotherapy. There is also a decreasing trend in HT-E curve of line 3 (green) because the 3rd location is near the 4th location. The rising and oscillation of HT-E curves of line 1 (blue) and line2 (red) indicate the small probability of tumor in these locations.

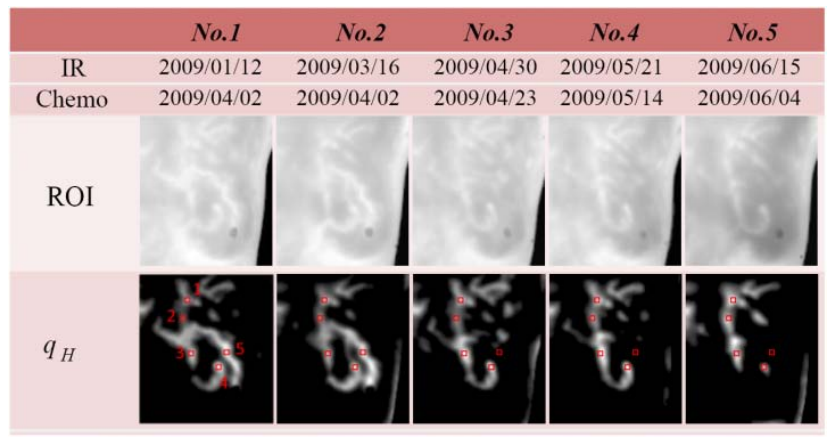

(a)

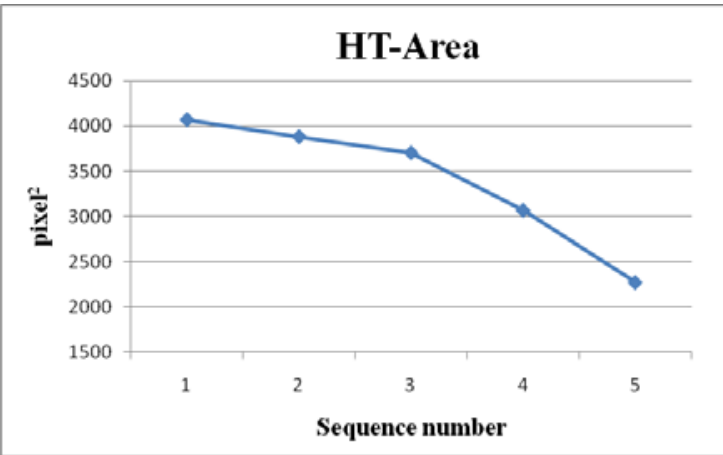

(b)

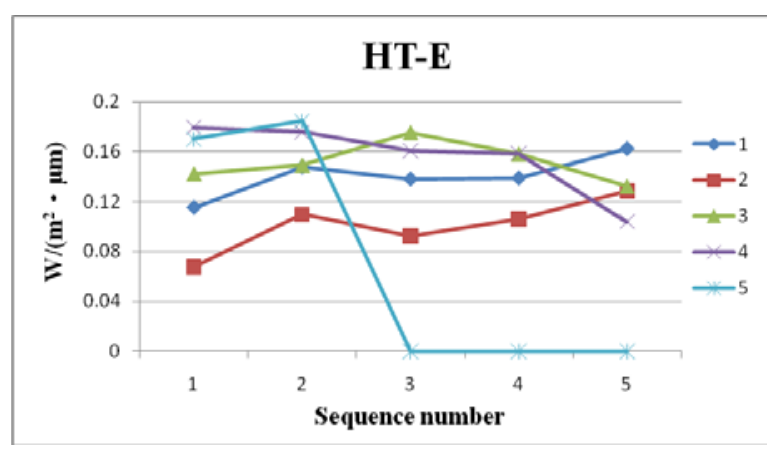

(c)

Fig. 4. (a) Results of the proposed approach for a sequence of chemotherapy, (b) HT-area curve, (c) HT-E curves of five selected ROIs

\section{CONCLUSION}

A QDS-IR system is proposed in this paper for quantitative assessment of chemotherapy. The proposed DS-HPS algorithm identifies the high temperature region, as well estimates the total energy of the attenuated LIR and MIR photons originated from the high temperature tissue and the normal temperature tissue. These two descriptors have been devised to quantify the change of the cancerous tissues, including the lesion size and peripheral vessel structures as well as the variation of the emitted energy. The result shows that the proposed longitudinal approach has the potential for quantitative assessment of chemotherapy.

\section{REFERENCES}

[1] Isard H. J., "Cancer in the "cold" breast thermogram," Am. J. Roentgenol., p. 793-796, 1976.

[2] Yang H. Q., Xie S. S., Lin Q. Y., et al., "A new infrared thermal imaging and its preliminary investigation of breast disease assessment," Complex Medical Engineering, p. 1071-1074, 2007.

[3] Keyserlingk J. R., Ahlgren P. D., Yu E., et al., "Infrared Imaging of the Breast: Initial Reappraisal Using HighResolution Digital Technology in 100 Successive Cases of Stage I and II Breast Cancer," The Breast Journal, p. 245-251, 1998.

[4] Cunningham L., "The anatomy of the arteries and veins of the breast," Journal of Surgical Oncology, p. 71-85, 1977.

[5] Lawson R., "Implications of surface temperatures in the diagnosis of breast cancer," Canadian Medical Association Journal, p. 309-310, 1956.

[6] Lawson R. N., Chughtai M. S., "Breast Cancer and Body Temperature," Canadian Medical Association Journal, p. 68-70, 1963.

[7] Stark A. M., Way S., "The use of thermovision in the detection of early breast cancer," Cancer, p. 1664-1670, 1974. 
[8] Zylberberg B., Salat-Baroux J., Ravina J. H., et al., "Initial chemoimmunotherapy in inflammatory carcinoma of the breast," Cancer, p. 1537-1543, 1982.

[9] Ng E. Y. K., Fok S. C., Peh Y. C., et al., "Computerized detection of breast cancer with artificial intelligence and thermograms," Journal of Medical Engineering \& Technology, p. 152 -157, 2002.

[10] Szu H., Miao L. and Qi H., "Thermodynamic free-energy minimization for unsupervised fusion of dual-color infrared breast images," SPIE, Florida, 2006.

[11] Yin F. F., Giger M. L., Doi K., et al, "Computerized detection of masses in digital mammograms: automated alignment of breast images and its effect on bilateral-subtraction technique," Med Phys, p. 445-452, 1994. 